\title{
ÉTIENNE GHYS
}

\section{Feuilletages holomorphes de codimension un sur les espaces homogènes complexes}

\author{
Annales de la faculté des sciences de Toulouse $6^{e}$ série, tome $5, \mathrm{n}^{\circ} 3$ \\ (1996), p. 493-519 \\ $<$ http://www.numdam.org/item?id=AFST_1996_6_5_3_493_0>
}

(C) Université Paul Sabatier, 1996, tous droits réservés.

L'accès aux archives de la revue «Annales de la faculté des sciences de Toulouse » (http://picard.ups-tlse.fr/ annales/) implique l'accord avec les conditions générales d'utilisation (http://www.numdam.org/conditions). Toute utilisation commerciale ou impression systématique est constitutive d'une infraction pénale. Toute copie ou impression de ce fichier doit contenir la présente mention de copyright.

\section{Numdam}

Article numérisé dans le cadre du programme Numérisation de documents anciens mathématiques http://www.numdam.org/ 


\title{
Feuilletages holomorphes de codimension un sur les espaces homogènes complexes ${ }^{(*)}$
}

\author{
ÉtIENNE Ghys ${ }^{(1)}$
}

\begin{abstract}
RÉSUMÉ. - Nous donnons une description qualitative assez précise des feuilletages holomorphes de codimension 1 sur les espaces homogènes complexes compacts. Après avoir donné une classification complète dans le cas des variétés kählériennes compactes, nous abordons le cas général en étudiant la position du feuilletage par rapport à la fibration de Tits.
\end{abstract}

Abstract - We give a rather precise description of the qualitative behaviour of holomorphic codimension 1 foliations on complex compact homogeneous spaces. First, we classify these foliations on Kähler homogeneous manifolds. Then, we deal with the general case by studying the relative position of the foliation and the Tits fibration.

\section{Introduction}

L'étude des propriétés qualitatives globales des feuilletages holomorphes non singuliers et de codimension 1 sur les variétés complexes compactes est assez peu développée. Dans ce travail, nous proposons une description assez précise de ces feuilletages sur les variétés homogènes compactes. Puisque cette classe de variétés est relativement riche, nous espérons ainsi obtenir quelques indications sur le comportement du feuilletage holomorphe de codimension 1 le plus général.

Le premier cas à considérer est bien sûr celui d'un tore complexe, $T=\mathbb{C}^{n} / \Lambda$, quotient de $\mathbb{C}^{n}$ par un réseau $\Lambda$. Les premiers exemples de

(*) Reçu le 11 janvier 1995

(1) Unité de Mathématiques Pures et Appliquées, U.M.R. 128 du C.N.R.S.

Ecole Normale Supérieure de Lyon, 46 allée d'Italie, F-69364 Lyon Cedex 07 (France)

E-mail : ghys@umpa.ens-lyon.fr 
feuilletages holomorphes de codimension 1 sur $T$ sont linéaires. On considère le feuilletage de $\mathbb{C}^{n}$ par les hyperplans parallèles à une direction donnée, qui passe évidemment au quotient sur $T$. On remarquera que ces feuilletages sont caractérisés par le fait qu'ils sont invariants par toutes les translations de $T$. Ils sont définis par une forme holomorphe (fermée) et toutes leurs feuilles sont sans holonomie.

Supposons maintenant qu'il existe une forme linéaire $\pi: \mathbb{C}^{n} \rightarrow \mathbb{C}$ qui envoie $\Lambda$ sur un réseau $\Lambda^{\prime}$ de $\mathbb{C}$. On note encore $\pi$ la projection correspondante de $T$ sur la courbe elliptique $C=\mathbb{C} / \Lambda^{\prime}$. Soit $u$ une fonction méromorphe non constante sur cette courbe elliptique et $\omega$ une forme holomorphe sur $T$ (qui est donc fermée à coefficients constants). La forme méromorphe $\Omega=\pi^{*}(u \mathrm{~d} z)+\omega$ est fermée et définit donc un feuilletage holomorphe en dehors du diviseur de ses pôles, image réciproque par $\pi$ des pôles de $u$. Nous affirmons que ce feuilletage se prolonge en fait en un feuilletage holomorphe non singulier sur le tore $T$ tout entier. Si $\omega$ s'annule sur les fibres de $\pi$, le feuilletage considéré est bien sûr celui défini par ces fibres. Sinon, quitte à choisir convenablement les coordonnées $\left(z_{1}, \ldots, z_{n}\right)$ dans $\mathbb{C}^{n}$, la forme $\Omega$, relevée à $\mathbb{C}^{n}$, s'écrit :

$$
u\left(z_{1}\right) \mathrm{d} z_{1}+\mathrm{d} z_{2} .
$$

Au voisinage d'un pôle $\alpha$ de $u$, on écrit :

$$
u\left(z_{1}\right)=\frac{1}{\left(z_{1}-\alpha\right)^{k}} v\left(z_{1}\right)
$$

avec $k \geq 1$ et $v$ holomorphe avec $v(\alpha) \neq 0$. Le feuilletage a donc aussi l'équation locale :

$$
v\left(z_{1}\right) \mathrm{d} z_{1}+\left(z_{1}-\alpha\right)^{k} \mathrm{~d} z_{2},
$$

qui se prolonge bien en une forme holomorphe non singulière en $z_{1}=0$. Les images réciproques par $\pi$ des pôles de $u$ sont des feuilles compactes. Les autres feuilles sont sans holonomie. Il s'agit d'une version complexe du tourbillonnement de Reeb. On remarquera que ces exemples sont invariants par les translations par les éléments du noyau de $\pi$, qui est un sous-tore de codimension 1 . Notons aussi qu'une application $\pi$ comme ci-dessus n'existe pas pour un réseau générique.

THÉORÈME 1.1. - Tout feuilletage holomorphe non singulier et de codimension 1 sur un tore complexe est de l'un des deux types précédents. 
Feuilletages holomorphes de codimension un sur les espaces homogènes complexes

Rappelons qu'un théorème de $\mathrm{A}$. Borel et $\mathrm{R}$. Remmert affirme qu'une variété kählérienne compacte homogène est le produit d'une variété généralisée de drapeaux $D$ et d'un tore $T$ (sect. 2). Sur le produit $\mathbb{C P}^{1} \times T$ d'une droite projective et d'un tore, on peut faire une construction de tourbillonnement analogue à celle que nous venons de décrire. Notons $\pi_{1}$ et $\pi_{2}$ les projections sur les premier et second facteurs de $\mathbb{C P}^{1} \times T$. Si $\omega$ est une forme holomorphe non nulle sur $T$ et si $u$ est une forme méromorphe (i.e. rationnelle) sur $\mathbb{C P}^{1}$, la forme fermée méromorphe $\pi_{1}^{*}(\omega)+\pi_{2}^{*}(u)$ sur $\mathbb{C P}^{1} \times T$ définit un feuilletage holomorphe non singulier.

THÉORÈME 1.2. - Soit $M=D \times T$ le produit d'une variété généralisée de drapeaux et d'un tore complexe. Un feuilletage holomorphe non singulier de codimension 1 sur $M$ peut être de trois types:

- le produit par $D$ d'un feuilletage holomorphe de $T$;

- si $D$ est de la forme $D^{\prime} \times \mathbb{C P}^{1}$, le produit par $D^{\prime}$ d'un feuilletage de $\mathbb{C P}^{1} \times T$ construit par tourbillonnement comme décrit plus haut;

- si $D$ est de la forme $D^{\prime} \times \mathbb{C P}^{1}$, le produit par $D^{\prime} \times T$ du feuilletage par points de $\mathbb{C P}^{1}$.

Considérons maintenant les variétés homogènes de la forme $G / \Gamma$ où $G$ est un groupe de Lie complexe et $\Gamma$ est un sous-groupe discret cocompact. Ces variétés compactes sont exactement celles dont le fibré tangent est holomorphiquement trivial et elles ne sont kählériennes que si le groupe $G$ est abélien (sect. 2). Nous décrirons à la section 4 les feuilletages holomorphes de codimension 1 sur ces variétés, mais nous nous contenterons dans cette introduction d'énoncer un résultat particulier. Si $\mathcal{H}$ est un sousespace de l'algèbre de Lie $\mathcal{G}$ de $G$, il définit un champ de plans sur $G$ invariant par translations à droite, qui passe donc au quotient sur $G / \Gamma$. Nous dirons qu'un tel champ de plans est homogène; il est intégrable si et seulement si $\mathcal{H}$ est une sous-algèbre. Les exemples ainsi obtenus peuvent avoir une dynamique assez compliquée. Rappelons qu'on dit qu'un groupe de Lie est parfait s'il coïncide avec son premier groupe des commutateurs.

Proposition 1.3. - Soit $G$ un groupe de Lie complexe parfait et $\Gamma$ un sous-groupe discret cocompact. Tout champ de plans holomorphe sur $G / \Gamma$ est homogène.

Une variété homogène compacte complexe quelconque fibre au-dessus d'une variété généralisée de drapeaux avec des fibres de la forme $G / \Gamma$; 
c'est la fibration de Tits que nous rappellerons à la section 2. En étudiant la position d'un feuilletage holomorphe par rapport à cette fibration et en utilisant la connaissance des feuilletages sur les variétés de drapeaux et les $G / \Gamma$, on obtient une bonne description des feuilletages sur les variétés homogènes quelconques. Nous nous contenterons ici d'énoncer quelques propriétés très générales. Rappelons qu'on dit qu'un feuilletage est transversalement projectif s'il peut être défini par des submersions locales dans la droite projective $\mathbb{C P}{ }^{1}$ telles que les changements de cartes transverses soient projectifs.

THÉORÈME 1.4. - Considérons un feuilletage holomorphe non singulier et de codimension 1 sur une variété complexe compacte homogène. Alors, il n'existe qu'un nombre fini de feuilles compactes à moins que toutes les feuilles ne soient compactes. Dans l'ouvert $U$ des feuilles non compactes, le feuilletage est transversalement projectif. Les adhérences des feuilles dans $U$ sont des sous-variétés qui sont toutes de codimension réelle 0,1 ou 2 . Toutes les feuilles compactes sont adhérentes à toutes les feuilles non compactes.

Comme il est bien connu, les théories des systèmes dynamiques réels et complexes de dimension 1 sont très proches [13]. On peut donc espérer obtenir pour les feuilletages holomorphes de codimension 1 des résultats analogues à ceux de la théorie classique des feuilletages de codimension réelle 1. Rappelons quelques-uns de ces résultats. Pour plus de détails, on pourra consulter [5].

Un ensemble minimal dans une variété feuilletée est un fermé non vide qui est réunion de feuilles et minimal pour ces propriétés. Si la variété ambiante est compacte, un tel ensemble minimal existe toujours. Un résultat fondamental de la théorie des feuilletages de codimension réelle 1 sur les variétés compactes est que la réunion des minimaux est fermée. D'autre part, si le feuilletage considéré est de classe $C^{2}$, tout ouvert saturé d'une variété compacte feuilletée de codimension réelle 1 contient des ensembles minimaux et leur réunion est un fermé (dans l'ouvert considéré). Ceci permet de définir par récurrence une suite de fermés $\mathcal{M}_{i}$ dans la variété ambiante : $\mathcal{M}_{0}$ est la réunion des ensembles minimaux de la variété et $\mathcal{M}_{i+1}-\mathcal{M}_{i}$ est la réunion des minimaux de la restriction du feuilletage au complémentaire de $\mathcal{M}_{i}$. Les feuilles de $\mathcal{M}_{i+1}-\mathcal{M}_{i}$ sont dites de hauteur $i+1$. Enfin, on dit que le feuilletage est de hauteur $\leq i$ si toutes ses feuilles sont de hauteur $\leq i$. Il existe des feuilletages de classe $C^{\infty}$ sur des variétés compactes qui sont de hauteur infinie mais ce phénomène ne se produit pas pour les feuilletages analytiques réels. 
Feuilletages holomorphes de codimension un sur les espaces homogènes complexes

On peut se demander s'il existe une théorie similaire pour les feuilletages holomorphes de codimension 1. Les résultats de cet article vont dans cette direction puisqu'ils signifient essentiellement que les feuilletages condidérés sont de hauteur 0 ou 1 . Certes, certains feuilletages holomorphes ont un comportement plus compliqué que celui que nous rencontrons ici. Par exemple, par suspension de groupes kleinéens, on obtient des feuilletages holomorphes de codimension 1 (sur des variétés non homogènes) qui possèdent des feuilles exceptionnelles, c'est-à-dire dont l'adhérence rencontre une transversale sur un ensemble de Cantor. Nous ne connaissons cependant aucun exemple de feuilletage holomorphe de codimension 1 sur une variété compacte dont la réunion des minimaux ne soit pas fermée, ni même d'exemple dont la hauteur soit strictement supérieure à 1 ... Comme motivation supplémentaire pour ces questions, on remarquera l'analogie avec la décomposition ensemble limite/domaine de discontinuité pour les groupes kleinéens ou la décomposition ensemble de Julia/ensemble de Fatou pour les fractions rationnelles. On pourra aussi consulter [8] pour certaines propriétés générales des feuilletages algébriques.

Cet article est organisé de la façon suivante. La section 2 contient quelques rappels classiques sur la géométrie des variétés complexes homogènes. Le théorème décrivant les feuilletages des tores est démontré à la section 3 . Le cas des variétés kählériennes est traité à la section 4 . Les sections 5 et 6 sont consacrées aux espaces homogènes du type $G / \Gamma$ avec $\Gamma$ discret. Enfin, le cas général est décrit à la section 7 dans laquelle nous détaillons quelques exemples concrets, tels que les variétés de Hopf.

\section{Rappels sur les variétés homogènes compactes complexes}

Pour une description des variétés homogènes complexes et une bibliographie, le lecteur pourra consulter [6].

Commençons par rappeler un fait extrêmement général de géométrie analytique, dû à A. Blanchard.

Proposition 2.1. - Soit $\Phi: X \rightarrow Y$ une application holomorphe propre entre deux variétés holomorphes. Soit par ailleurs $G$ un groupe topologique connexe agissant sur $X$ par biholomorphismes. Alors, l'action de $G$ permute les composantes connexes des fibres de $\Phi$.

Démonstration. - Soit $y \in Y$ et $K$ une composante de $\Phi^{-1}(y)$. Soit $U$ un voisinage de $y$ biholomorphe à un ouvert de $\mathbb{C}^{k}$. Soit $g \in G$ suffisamment 
proche de l'identité pour que l'image $g \cdot K$ de $K$ par $g$ soit contenue dans $\Phi^{-1}(U)$. Alors $\Phi(g \cdot K)$ est un ensemble analytique compact contenu dans $U$, donc réduit à un point par le principe du maximum. Ceci signifie précisément que l'image de $K$ par $g$ est contenue dans une fibre de $\Phi$. Par connexité de $G$, on établit la même propriété pour tous les éléments de $G$, c'est-à-dire la proposition.

Corollaire 2.2. - Soit $G / H$ une variété complexe compacte homogène, quotient d'un groupe de Lie complexe connexe $G$ par un sous-groupe de Lie complexe fermé $H$. Soit $\Phi: G / H \rightarrow Y$ une application holomorphe vers une variété holomorphe $Y$. Alors il existe un sous-groupe complexe fermé $\widetilde{H}$ contenant $H$ et une application holomorphe $\Phi^{\prime}: G / \widetilde{H} \rightarrow Y$ tels que :

- $\Phi=\Phi^{\prime} \circ \pi$ où $\pi$ désigne la projection de $G / H$ sur $G / \widetilde{H}$;

- les fibres de $\Phi^{\prime}$ sont finies.

Démonstration. - En appliquant la proposition 2.1 à l'application $\Phi$, on voit que l'action (transitive) de $G$ sur l'espace homogène $G / H$ permute les composantes connexes des fibres de $\Phi$. Soit $\widetilde{H}$ le sous-groupe de $G$ stabilisant la composante connexe de la fibre contenant le point base de $G / H$. L'espace homogène $G / \widetilde{H}$ est l'espace des composantes connexes des fibres de $\Phi$ de sorte que le corollaire résulte du fait qu'une fibre n'a qu'un nombre fini de composantes.

La manière la plus simple de décrire une variété compacte homogène est d'utiliser la fibration de Tits.

THÉORÈME 2.3 (Tits). - Soit $M=G / H$ une variété homogène complexe compacte connexe. Il existe un sous-groupe de Lie complexe fermé $\widetilde{H}$ de $G$, contenant $H$, tel que les fibres de la projection naturelle de $G / H$ sur $G / \widetilde{H}$ soient des espaces homogènes de la forme $N / \Gamma$ où $N$ est un groupe de Lie complexe connexe et $\Gamma$ un sous-groupe discret cocompact. De plus, la variété $G / \widetilde{H}$ est algébrique projective.

Démonstration. - Soit $H_{0}$ la composante neutre de $H$ et $\widetilde{H}$ le normalisateur de $H_{0}$, contenant évidemment $H$. Soit $\operatorname{Ad}: G \rightarrow \operatorname{Aut}(\mathcal{G})$ la représentation adjointe de $G$ dans son algèbre de Lie $\mathcal{G}$. Le sous-groupe $\widetilde{H}$ est l'image réciproque par $\operatorname{Ad}$ du sous-groupe $\operatorname{de} \operatorname{Aut}(\mathcal{G})$ qui préserve la sous-algébre de Lie $\mathcal{H}_{0}$. L'espace homogène $G / \widetilde{H}$ s'identifie à l'orbite de $\mathcal{H}_{0}$ sous l'action 
Feuilletages holomorphes de codimension un sur les espaces homogènes complexes

de $\operatorname{Ad}(G)$ dans la grassmannienne des sous-espaces de $\mathcal{G}$; c'est donc une variété projective. Enfin, les fibres de la projection de $G / H$ sur $G / \widetilde{H}$ sont des espaces homogènes de la forme $\widetilde{H} / H$. Puisque $H_{0}$ est normal dans $\widetilde{H}$, on peut aussi décrire ces fibres comme $\left(\widetilde{H} / H_{0}\right) /\left(H / H_{0}\right)$, c'est-à-dire sous la forme annoncée $N / \Gamma$ puisque $\Gamma=H / H_{0}$ est un sous-groupe discret de $N=\widetilde{H} / H_{0}$.

La base $D=G / \widetilde{H}$ de la fibration de Tits est une variété de drapeaux généralisée. C'est bien sûr un espace homogène sous l'action de $G$, mais l'action du radical $R$ de $G$ possède un point fixe par le théorème de point fixe de Borel et, puisque $R$ est normal dans $G$, l'action de $R$ est en fait triviale. Autrement dit, $D$ peut aussi s'écrire sous la forme $S / P$ où $S$ est un groupe semi-simple complexe. En utilisant à nouveau le théorème de Borel, on voit que $P$ contient un sous-groupe résoluble maximal de $G$; on dit que $P$ est un sous-groupe parabolique.

La fibre $N / \Gamma$ de la fibration de Tits est holomorphiquement parallélisable. Le résultat suivant est bien connu.

THÉORÈME 2.4 (Wang). - Soit $\Gamma$ un sous-groupe discret cocompact d'un groupe de Lie complexe connexe $N$. La variété $N / \Gamma$ est kählérienne (si et) seulement si $N$ est abélien.

Démonstration. - Toute forme linéaire sur l'algèbre de Lie de $N$ donne une 1-forme différentielle holomorphe invariante à droite sur $N$ et donc une 1 -forme holomorphe sur $N / \Gamma$. Si cette variété est kählérienne et compacte, toutes les formes holomorphes sont fermées. Une 1-forme invariante à droite sur $N$ est fermée si et seulement si elle s'annule sur les crochets de champs invariants à droite. Il en résulte bien que si $N / \Gamma$ est kählérienne le groupe $N$ est abélien.

Enfin, nous utiliserons le résultat suivant que nous avons déjà mentionné.

THÉORÈME 2.5 (Borel-Remmert). - Toute variété kählérienne compacte homogène est le produit direct d'un tore complexe et d'une variété de drapeaux.

Démonstration (esquisse). - La fibration de Tits et la proposition 2.4 montrent qu'une telle variété fibre au-dessus d'une variété de drapeaux avec une fibre qui est un tore complexe. Il s'agit de démontrer que cette fibration est triviale. Pour cela, on utilise l'application d'Albanese. Rappelons que si $M$ est une variété kählérienne compacte, l'intégration des 1-formes 


\section{Étienne Ghys}

holomorphes (fermées) définit une application holomorphe de $M$ à valeurs dans le tore complexe, quotient du groupe de cohomologie $H^{1}(M ; \mathbb{C})$ par le réseau des classes entières. Dans le cas qui nous intéresse, l'application produit de l'application d'Albanese et de la fibration de Tits envoie la variété sur le produit d'une variété de drapeaux et d'un tore. On s'assure qu'il s'agit d'un biholomorphisme.

\section{Feuilletages holomorphes sur les tores}

Dans cette section, nous démontrons le théorème 1.1, première étape dans notre description des feuilletages des variétés homogènes générales. Dans la suite de cet article, tous les feuilletages sont non singuliers.

Nous commençons par une conséquence facile de 2.2. Soit $T$ un tore complexe, quotient de $\mathbb{C}^{n}$ par un réseau $\Lambda$.

Proposition 3.1.- Soit $\mathcal{P}$ un champ d'hyperplans holomorphe sur $T$. Alors, il existe un sous-tore de $T$ non trivial formé de translations qui préservent $\mathcal{P}$.

Démonstration. - Le fibré tangent à $T$ est bien sûr trivialisé holomorphiquement de sorte que $\mathcal{P}$ correspond à une application holomorphe $\Phi$ de $T$ vers l'espace des hyperplans de $\mathbb{C}^{n}$, c'est-à-dire vers l'espace projectif $\mathbb{C P}^{n-1}$. Les fibres génériques de $\Phi$ sont donc de dimension supérieure ou égale à 1 . D'après le corollaire 2.2 , cette application $\Phi$ transite à travers un quotient non trivial de $T$ qui est encore un espace homogène d'un groupe abélien, c'est-à-dire un tore quotient. C'est précisément l'énoncé de la proposition qu'il fallait démontrer.

Soit maintenant $\mathcal{F}$ un feuilletage holomorphe de codimentsion 1 sur $T$. Notons $T_{1}$ la composante neutre du sous-groupe de $T$ formé des translations qui préservent $\mathcal{F}$. Nous savons que $T_{1}$ est non trivial et nous allons utiliser l'intégrabilité de $\mathcal{F}$ pour montrer que $T_{1}$ est en fait de dimension supérieure ou égale à $n-1$. On note $C$ le quotient de $T$ par $T_{1}$ et $\pi: T \rightarrow C$ la projection canonique.

Proposition 3.2.- Si $\mathcal{F}$ est transverse aux fibres de $\pi$, alors $T_{1}=T$, c'est-à-dire que $\mathcal{F}$ est un feuilletage linéaire de $T$.

Démonstration. - La trace de $\mathcal{F}$ sur une fibre $\pi^{-1}(x)$ est un champ de plans invariant par les translations de $T_{1}$, c'est-à-dire un feuilletage linéaire 
Feuilletages holomorphes de codimension un sur les espaces homogènes complexes

de $T_{1}$. Si un feuilletage est transverse aux fibres compactes d'une fibration, toutes les traces sur ces fibres sont isotopes (par une isotopie de classe $C^{\infty}$ ) (voir aussi $\S 5.4$ ). Puisque deux feuilletages linéaires de codimension complexe 1 sur $T$ ne peuvent être $C^{\infty}$-isotopes que s'ils coïncident, il en résulte que la trace de $\mathcal{F}$ sur $\pi^{-1}(x) \simeq T_{1}$ est un feuilletage linéaire de $T_{1}$ indépendant de $x$. On peut donc trouver un champ de vecteurs holomorphe $X$ sur $T$, tangent aux fibres de $\pi$, qui est transverse à $\mathcal{F}$. Soit $\Omega$ la 1 -forme holomorphe nulle sur $\mathcal{F}$ et telle que $\Omega(X)=1$. Cette forme holomorphe est nécessairement à coefficients constants, c'est-à-dire $\mathcal{F}$ est un feuilletage linéaire.

Lemme 3.3.- Si une fibre de $\pi$ n'est pas transverse à $\mathcal{F}$, elle est contenue dans une feuille de $\mathcal{F}$.

Démonstration. - Puisque $\mathcal{F}$ est invariant par les translations de $T_{1}$, une fibre non transverse à $\mathcal{F}$ en un certain point est non transverse partout, c'est-à-dire est partout tangente à $\mathcal{F}$.

Proposition 3.4. - Il existe au moins une fibre $\pi^{-1}(x)$ transverse à $\mathcal{F}$.

Démonstration. - Dans le cas contraire, toutes les fibres seraient contenues dans une feuille de $\mathcal{F}$, c'est-à-dire que $\mathcal{F}$ serait l'image réciproque par $\pi$ d'un certain feuilletage $\mathcal{F}^{\prime}$ de $C$. En appliquant la proposition 3.1 à $\mathcal{F}^{\prime}$, on obtiendrait un sous-tore de $T$ contenant strictement $T_{1}$ et préservant $\mathcal{F}$, contredisant ainsi la définition de $T_{1}$.

Nous allons maintenant étudier le cas où l'ensemble $\Sigma$ des points $x$ de $C$ tels que $\pi^{-1}(x)$ n'est pas transverse à $\mathcal{F}$ est un ensemble analytique non vide et différent de $T$ tout entier.

Lemme 3.5. $-\pi^{-1}(\Sigma)$ est une réunion finie de feuilles compactes de $\mathcal{F}$.

Démonstration. - Si $x \in C$ n'est pas dans $\Sigma$, la trace de $\mathcal{F}$ sur $\pi^{-1}(x) \simeq T_{1}$ est un feuilletage invariant par translations de $T_{1}$; c'est donc un feuilletage linéaire de $T_{1}$. Par un argument que nous avons déjà utilisé, ce feuilletage linéaire ne dépend pas de $x$ (on remarquera que $T \backslash \Sigma$ est connexe). Il existe donc un champ de vecteurs holomorphe $X$ sur $T$, tangent à $T_{1}$ et transverse à $\mathcal{F}$ sur $\pi^{-1}(C \backslash \Sigma)$. Soit $\Omega$ la 1 -forme méromorphe sur $T$ qui est nulle sur le feuilletage et qui est telle que $\Omega(X)=1$. Comme les translations de $T_{1}$ préservent $\mathcal{F}$, la dérivée de Lie $\mathcal{L}_{X}(\Omega)=i_{X} \mathrm{~d} \Omega+\mathrm{d} i_{X} \Omega$ est nulle. Puisque $\Omega(X)=1$, c'est que $\Omega$ est fermée. 
LEMME 3.9. - Les sous-tores $T_{1}^{\prime}$ et $T_{1}$ coïncident.

Démonstration. - Soit $Y$ un champ de vecteurs holomorphe sur $T$. Les pôles de la fonction méromorphe $\Omega(Y)$ sont dans $\pi^{-1}(\Sigma)$. Il en résulte que $\Omega(Y)$ est holomorphe sur toutes les fibres $\pi^{\prime-1}(x) \simeq T_{1}^{\prime}$ avec $x$ dans $C^{\prime}$ différent des $\alpha_{i}(i=1, \ldots, k)$, donc constante. Cela signifie que $\Omega$ et donc $\mathcal{F}$ sont invariantes par toutes les translations de $T_{1}^{\prime}$ et donc que $T_{1}^{\prime}=T_{1}$.

Le théorème 1.1 est maintenant facile. Nous venons de voir que pour tout champ de vecteurs holomorphe $Y$ sur $T$, la fonction $\Omega(Y)$ est constante sur les fibres de $\pi$, c'est-à-dire qu'elle provient d'une fonction méromorphe sur la courbe elliptique $C$. Choisissons les coordonnées $\left(z_{1}, \ldots, z_{n}\right)$ dans $\mathbb{C}^{n}$ de telle sorte que $T_{1}$ soit la projection de $\{0\} \times \mathbb{C}^{n-1}$. La forme $\Omega$, relevée dans $\mathbb{C}^{n}$, s'écrit sous la forme:

$$
u\left(z_{1}\right) \mathrm{d} z_{1}+a_{2}\left(z_{1}\right) \mathrm{d} z_{2}+\cdots+a_{n}\left(z_{1}\right) \mathrm{d} z_{n} .
$$

La forme $\Omega$ étant fermée, les fonctions $a_{2}, \ldots, a_{n}$ se réduisent à des constantes. Nous avons bien montré que le feuilletage $\mathcal{F}$ est défini par une forme méromorphe $\Omega$ du type $\pi^{*}(u \mathrm{~d} z)+\omega$ où $u$ est une fonction méromorphe sur $C$ et $\omega$ est une forme holomorphe sur $T$. C'est précisément l'énoncé du théorème 1.1.

Dominique Cerveau m'a indiqué une autre démonstration du théorème 1.1 qui repose cependant sur les mêmes idées.

\section{Feuilletages sur les variétés kählériennes}

Dans cette section, nous démontrons le théorème 1.2 décrivant les feuilletages holomorphes de codimension 1 sur les variétés kählériennes homogènes compactes.

Commençons par étudier le cas d'une variété de drapeaux.

THÉORÈme 4.1. - Soit $D$ une variété de drapeaux. S'il existe un feuilletage holomorphe sur $D$ de codimension 1 , alors $D$ peut se décomposer en un produit $D^{\prime} \times \mathbb{C P}^{1}$ et le feuilletage est le feuilletage produit.

Nous utiliserons trois lemmes.

LEMme 4.2. - La première classe de Chern du fibré normal à un feuilletage holomorphe d'une variété de drapeaux $D$ ne peut être triviale. 
Feuilletages holomorphes de codimension un sur les espaces homogènes complexes

Démonstration. - On remarque d'abord que les variétés de drapeaux sont simplement connexes de sorte que leur cohomologie à coefficients entiers est sans torsion. En particulier, l'annulation d'une classe de Chern rationnelle entraîne l'annulation de la classe entière. Par ailleurs, puisque le premier groupe de cohomologie de la variété algébrique projective $D$ est nul, les fibrés en droites sur $D$ sont caractérisés par leur première classe de Chern entière. Si cette classe était nulle, le feuilletage serait défini par une 1-forme holomorphe non singulière sur $D$. Ceci est impossible car il n'y a pas de 1-forme holomorphe non nulle sur $D$ (toujours car le premier groupe de cohomologie est nul).

LEMME 4.3. - Le carré de la première classe de Chern d'un feuilletage holomorphe de codimension 1 est nul dans la cohomologie à coefficients rationnels.

Démonstration. - C'est un exemple élémentaire d'application du théorème d'annulation de Bott, dans le cas des feuilletages holomorphes [1].

Lemme 4.4. - Soit D une variété de drapeaux. Supposons qu'il existe une classe non nulle $c$ de $H^{2}(D ; \mathbb{Q})$ dont le carré est nul. Alors $D$ est un produit $D^{\prime} \times \mathbb{C P}^{1}$ et $c$ est un multiple (de l'image dans $H^{2}(D ; \mathbb{Q})$ ) du générateur de $H^{2}\left(\mathbb{C P}^{1} ; \mathbb{Q}\right)$.

Démonstration. - Écrivons $D$ sous la forme $G / P$ où $G$ est un groupe semi-simple complexe et $P$ est un sous-groupe parabolique, c'est-à-dire contenant un sous-groupe de Borel $B$ (sous-groupe résoluble maximal). Rappelons que la projection de $G / B$ sur $G / P$ induit une injection en cohomologie, surjective au niveau du second groupe de cohomologie (c'est un exemple du splitting principle [2]). La cohomologie de $G / B$ à coefficients dans $\mathbb{C}$ est facile à décrire (voir par exemple [4]) : soit $\mathcal{H}$ une sous-algèbre de Cartan de l'algèbre de Lie $\mathcal{G}$ de $G$ et $W$ le groupe de Weyl agissant sur $\mathcal{H}$. Alors l'anneau de cohomologie $H^{*}(G / B ; \mathbb{C})$ est le quotient de l'algèbre des polynômes sur $H$ par l'idéal engendré par les polynômes symétriques, c'està-dire invariants par $W$. Dans cette description, les classes de cohomologie correspondant aux éléments du dual de $\mathcal{H}$ sont de degré 2 .

Revenant au lemme, on peut donc envisager la classe $c$ comme une forme linéaire sur $\mathcal{H}$ dont le carré est une forme quadratique invariante par le groupe de Weyl. Supposons d'abord $\mathcal{G}$ simple. Comme il est bien connu, le groupe de Weyl préserve alors (à une constante près) une unique forme quadratique : la forme de Killing (qui est non dégénérée). Celle-ci 


\section{Étienne Ghys}

ne peut être le carré d'une forme linéaire que si $\mathcal{H}$ est de dimension 1 , c'est-à-dire si $G$ est (localement) isomorphe à $\mathrm{SL}(2, \mathbb{C})$. Lorsque l'algèbre $\mathcal{G}$ n'est que semi-simple, les formes quadratiques invariantes par $W$ sont les combinaisons linéaires des formes de Killing des facteurs simples. Une telle forme quadratique ne peut être un carré que si elle provient d'un facteur simple (localement) isomorphe à $\mathrm{SL}(2, \mathbb{C})$. À conjugaison près, le seul sousgroupe parabolique de $\mathrm{SL}(2, \mathbb{C})$ est le sous-groupe des matrices triangulaires supérieures, correspondant à la variété des drapeaux $\mathbb{C P}^{1}$. Ainsi, $D$ s'écrit comme un produit $D^{\prime} \times \mathbb{C P}^{1}$ et $c$ est un multiple de la classe fondamentale du second facteur.

La preuve du théorème 4.1 est maintenant facile. Considérons en effet un feuilletage $\mathcal{F}$ holomorphe de codimension 1 sur une variété de drapeaux $D$. Il résulte des lemmes précédents que $D$ s'écrit $D^{\prime} \times \mathbb{C P}^{1}$ et il s'agit de montrer que les $D^{\prime} \times\{*\}$ sont contenus dans les feuilles. Le fibré normal à $\mathcal{F}$ est donc isomorphe à un multiple de l'image réciproque du fibré canonique au-dessus de $\mathbb{C P}^{1}$. Cela signifie que pour tout point $x$ de $\mathbb{C P}^{1}$, la restriction du feuilletage $\mathcal{F}$ à $D^{\prime} \times\left(\mathbb{C P}^{1} \backslash\{x\}\right)$ est définie par une forme holomorphe. Puisqu'une telle forme s'annule évidemment sur $D^{\prime} \times\{y\}$ pour tout $y$, le feuilletage $\mathcal{F}$ coïncide bien avec le feuilletage produit, comme annoncé.

Nous abordons maintenant la preuve du théorème 1.2 qui décrit les feuilletages holomorphes $\mathcal{F}$ sur les variétés du type $D \times T$ où $D$ est une variété de drapeaux et $T$ un tore complexe. Nous suivrons une méthode très proche de celle que nous venons d'utiliser. Soit $c$ la première classe de Chern du fibré normal à $\mathcal{F}$.

LEMME 4.5.- Deux cas sont possibles:

- c est l'image réciproque d'une classe de $H^{2}(T ; \mathbb{Z})$ par la projection de $D \times T$ sur $T$;

- c est l'image réciproque d'un multiple de la classe fondamentale de $\mathbb{C P}^{1}$ dans une décomposition en produit $D^{\prime} \times \mathbb{C P}^{1} \times T$.

Démonstration. - Puisque le premier groupe de cohomologie de $D$ est nul, la formule de Künneth montre que $c$ peut s'écrire de manière unique comme une somme $c_{1}+c_{2}$ où $c_{1}$ est une classe qui provient de $H^{2}(D ; \mathbb{Z})$ et $c_{2}$ de $H^{2}(T ; \mathbb{Z})$. Le lemme 4.3 montre que les classes $c_{1}^{2}, c_{2}^{2}, c_{1} c_{2}$ sont nulles dans $H^{4}(D \times T ; \mathbb{Z})$. Il en résulte que $c_{1}$ ou $c_{2}$ est nulle. Si $c_{1}$ est nulle, le lemme 4.4 montre que $c_{2}$ provient d'un facteur $\mathbb{C P}^{1}$. 
Feuilletages holomorphes de codimension un sur les espaces homogènes complexes

Nous analysons maintenant chacun de ces deux cas suivants.

Lemme 4.6. - Si la classe c est l'image réciproque d'une classe de $H^{2}(T ; \mathbb{Z})$ par la projection de $D \times T$ sur $T$, alors le feuilletage $\mathcal{F}$ est l'image réciproque d'un feuilletage holomorphe de $T$ par la projection de $D \times T$ surT.

Démonstration. - La classe de Chern du fibré normal au feuilletage est l'image réciproque d'une classe entière du tore $T$. L'image réciproque du fibré normal dans le revêtement universel $D \times \mathbb{C}^{n}$ est alors un fibré en droites de classe de Chern triviale et donc un fibré holomorphiquement trivial (car $H^{1}(D ; \mathbb{Z})=0$ ). Autrement dit, le relevé de $\mathcal{F}$ à ce revêtement est défini par une forme holomorphe qui, comme nous l'avons déjà remarqué plusieurs fois, est nécessairement nulle en restriction aux $D \times\{x\}$. Cela signifie que $\mathcal{F}$ est tangent à ces $D \times\{x\}$ et donc qu'il est l'image réciproque d'un feuilletage de $T$.

LEMME 4.7. - Supposons que c soit l'image réciproque d'un multiple de la classe fondamentale de $\mathbb{C P}^{1}$ dans une décomposition en produit $D=$ $D^{\prime} \times \mathbb{C P}^{1} \times T$. Alors $\mathcal{F}$ est l'image réciproque d'un feuilletage de $\mathbb{C P}^{1} \times T$, invariant par les translations par $T$ agissant sur $D \times T$.

Démonstration. - Il s'agit d'abord de montrer que les $D^{\prime} \times\{*\} \times\{*\}$ sont contenus dans les feuilles. Soit $x$ un point de $\mathbb{C P}^{1}$. Le fibré normal à $\mathcal{F}$, relevé à $D \times \mathbb{C}^{n}$ et restreint à $D^{\prime} \times\left(\mathbb{C P}^{1} \backslash\{x\}\right) \times \mathbb{C}^{n}$ est trivial, de sorte que le même argument que précédemment montre bien que $\mathcal{F}$ est l'image réciproque d'un feuilletage de $\mathbb{C P}^{1} \times T$ par la projection de $D^{\prime} \times \mathbb{C P}^{1} \times T$ sur $\mathbb{C P}^{1} \times T$. On peut donc supposer que $D^{\prime}$ est trivial (i.e. réduit à un point).

La restriction du fibré tangent de $\mathbb{C P}^{1} \times T$ à $\{x\} \times T$ est un fibré holomorphiquement trivial de rang $n+1$ et le feuilletage $\mathcal{F}$ permet donc de définir une application holomorphe $\Phi_{x}$ de $\{x\} \times T$ vers la grassmannienne des hyperplans dans $\mathbb{C}^{n+1}$, c'est-à-dire vers un espace projectif $\mathbb{C P}^{n}$. Le fibré normal à $\mathcal{F}$, restreint à $\{x\} \times T$, est l'image réciproque du fibré tautologique sur $\mathbb{C P}^{n}$ par l'application holomorphe $\Phi_{x}$; il a donc une classe de Chern non nulle à moins que $\Phi_{x}$ ne soit constant. Puisque l'hypothèse du lemme entraîne évidemment que cette classe de Chern est nulle, on en déduit que toutes ces applications $\Phi_{x}$ sont constantes, c'est-à-dire que $\mathcal{F}$ est invariant par les translations de $T$ sur $\mathbb{C P P}^{1} \times T$.

La fin de la preuve du théorème 1.2 est maintenant facile. Si l'on dispose d'un feuilletage $\mathcal{F}$ sur $\mathbb{C P}^{1} \times T$ invariant par les translations par $T$, il suffit de 
procéder comme nous l'avons fait pour étudier le cas des tores. Si $\mathcal{F}$ n'est pas un feuilletage produit dont les feuilles sont les $\{x\} \times T$, alors il est transverse à tous les tores $\{x\} \times T$ sauf un nombre fini d'entre eux. Les traces sur ces tores transverses (identifiés à $T$ ) sont toutes le même feuilletage linéaire. En choisissant convenablement des coordonnées $\left(z_{1}, \ldots, z_{n}\right)$ dans $\mathbb{C}^{n}$ et $\zeta$ dans $\mathbb{C} \cup\{\infty\} \simeq \mathbb{C P}^{1}$, le feuilletage est donc défini par une forme méromorphe $\Omega$ de la forme :

$$
\Omega=\mathrm{d} z_{1}+u\left(\zeta, z_{1}, \ldots, z_{n}\right) \mathrm{d} \zeta
$$

où $u$ est méromorphe. Puisque $\mathcal{F}$ est invariant par translation, la fonction $u$ ne dépend en fait que de $\zeta$, c'est-à-dire que $u(\zeta) \mathrm{d} \zeta$ est une forme méromorphe (i.e. rationnelle) sur $\mathbb{C P P}^{1}$. Nous avons bien obtenu la description donnée dans le théorème 1.2.

\section{Feuilletages holomorphes sur $G / \Gamma$}

Soit $\Gamma$ un sous-groupe discret cocompact du groupe de Lie complexe connexe $G$ et $M=G / \Gamma$ la variété quotient. Les translations à droite de $G$ permettent de trivialiser le fibré tangent à $G$ et donc le fibré tangent à $M$. Soit $\mathcal{P}$ un champ de plans holomorphe sur $M$ (de dimension quelconque). Ceci définit une application holomorphe $\Phi$ de $M$ vers une grassmannienne $Y$ et on peut appliquer le corollaire $2.2:$ il existe un sous-groupe complexe fermé $\widetilde{H}$ contenant $\Gamma$ et une application holomorphe $\Phi^{\prime}: G / \widetilde{H} \rightarrow Y$ tels que :

- $\Phi=\Phi^{\prime} \circ \pi$ où $\pi$ désigne la projection de $G / \Gamma$ sur $G / \widetilde{H}$;

- les fibres de $\Phi^{\prime}$ sont finies.

On remarquera que $\widetilde{H}$ n'est pas nécessairement connexe.

LEMME 5.1. - Le sous-groupe $\widetilde{H}$ est distingué et contient le premier groupe dérivé de $G$. L'espace homogène $G / \widetilde{H}$ est un tore complexe.

Démonstration. - Puisque les fibres de $\Phi^{\prime}$ sont finies, la variété $G / \widetilde{H}$ est projective, donc kählérienne. Le théorème de Borel-Remmert 2.5 montre que $G / \widetilde{H}$ est le produit d'une variété de drapeaux $D$ et d'un tore $T$. Nous affirmons qu'en fait $G / \widetilde{H}$ est un tore. 
Rappelons un théorème de $\mathrm{D}$. Montgomery [12]. Soit $H_{0}$ un sous-groupe fermé d'un groupe de Lie $G$. Si $H_{0}$ possède un nombre fini de composantes connexes et si le quotient $G / H_{0}$ supporte une mesure finie invariante par $G$, alors l'action de $G$ transite à travers un groupe compact. Plus précisément, il existe un morphisme surjectif de $G$ sur un groupe compact dont le noyau est contenu dans $H_{0}$.

Revenons à notre situation. Puisque $D$ est une variété de drapeaux, on peut écrire $D=S / P$ où $S$ est un groupe semi-simple complexe, quotient de $G$, et $P$ est un sous-groupe parabolique connexe. Rappelons qu'un groupe de Lie qui possède un sous-groupe discret cocompact est nécessairement unimodulaire, c'est-à-dire que sa mesure de Haar est bi-invariante. L'action de $G$ sur $G / \Gamma$ préserve donc une mesure finie. L'image directe de cette mesure par projection sur $D$ est une mesure finie $G$-invariante et donc $S$-invariante. D'après le théorème de Montgomery, l'action de $S$ sur $D$ transite à travers un groupe compact, quotient de $S$. Un groupe semisimple complexe n'a pas de quotient compact non trivial de sorte que $D$ est nécessairement réduit à un point. Nous avons bien montré que $G / \widetilde{H}$ est un tore complexe. L'action de $G$ sur ce tore est nécessairement par translations de sorte qu'il existe un morphisme de $G$ sur un groupe abélien dont le noyau est $\widetilde{H}$. En particulier, $\widetilde{H}$ est distingué et contient le premier groupe dérivé de $G$.

La proposition 1.3 est facile : si le groupe $G$ est parfait, le lemme précédent montre que $\Phi$ est une application constante, c'est-à-dire que lorsque l'on relève le champ de plans $\mathcal{P}$ à $G$, on obtient un champ de plans constant par rapport au parallélisme, c'est-à-dire invariant à droite. C'est précisément le contenu de la proposition 1.3.

Nous supposerons maintenant que $\mathcal{P}$ est un champ d'hyperplans intégrable, c'est-à-dire un feuilletage holomorphe de codimension 1 . Toujours sous l'hypothèse que $G$ est parfait, on obtient donc que les feuilles de $\mathcal{F}$ sont les orbites d'un sous-groupe $F$ de codimension 1 dans $G$, agissant par translations à gauche sur l'espace homogène $G / \Gamma$. Rappelons que l'un des premiers résultats de $\mathrm{S}$. Lie est précisément la classification des sous-groupes fermés $F$ de codimension 1 des groupes de Lie $G$, c'est-à-dire des géométries de dimension 1. Trois cas sont possibles [9] :

1. géométrie de translation : il existe une surjection $\sigma$ de $G$ sur $\mathbb{C}$ et un sous-groupe discret de $\mathbb{C}$ dont l'image réciproque par $\sigma$ est le sousgroupe $F$. L'espace homogène $G / F$ est la droite complexe $\mathbb{C}$ (ou un quotient par un réseau) et l'action de $G$ est par translations; 
2. géométrie affine : il existe une surjection $\sigma$ de $G$ sur le groupe $\operatorname{Aff}(\mathbb{C})$ des bijections affines de $\mathbb{C}$ telles que $F$ est l'image réciproque par $\sigma$ du sous-groupe des homothéties de centre 0 . L'espace homogène $G / F$ est la droite complexe $\mathbb{C}$ et l'action de $G$ est affine;

3. géométrie projective : il existe une surjection $\sigma$ de $G$ sur $\operatorname{PSL}(2, \mathbb{C})$ telle que $F$ est l'image réciproque du sous-groupe des matrices triangulaires supérieures. L'espace homogène $G / F$ est la droite projective complexe $\mathbb{C P}^{1}$ et l'action de $G$ est projective.

Remarquons que si $G$ est parfait, il n'existe évidemment pas de sousgroupe de type 1 . Si $G$ est de plus semi-simple, il n'existe pas de surjection sur le groupe résoluble $\operatorname{Aff}(\mathbb{C})$. Si $G$ est un groupe simple, une surjection sur $\operatorname{PSL}(2, \mathbb{C})$ ne peut exister que s'il s'agit d'un isomorphisme. Plus généralement, si $G$ est un groupe de Lie semi-simple sans facteur simple (localement) isomorphe à $\operatorname{PSL}(2, \mathbb{C})$, alors il ne possède aucun sous-groupe de codimension 1 . On obtient donc comme exemple d'application la proposition suivante.

Proposition 5.2.- Soit $G$ un groupe de Lie complexe semi-simple sans facteur localement isomorphe à $\operatorname{PSL}(2, \mathbb{C})$ et $\Gamma$ un sous-groupe discret cocompact. Alors il n'existe aucun feuilletage holomorphe de codimension 1 $\operatorname{sur} G / \Gamma$.

Reprenons l'étude du cas général où $G$ n'est plus supposé parfait. On dispose donc d'une fibration $\pi: G / \Gamma \rightarrow G / \widetilde{H}=T$ au-dessus d'un tore. Par définition, les espaces tangents à $\mathcal{F}$ aux divers points d'une fibre de $\pi$ sont les mêmes, par rapport à la trivialisation du fibré tangent à $G / \Gamma$. Autrement dit, le relevé de $\mathcal{F}$ à $G$ est invariant par les translations à droite par $\widetilde{H}$. En particulier, si $\mathcal{F}$ est transverse à une fibre de $\pi$ en un point, il est transverse en tous les points de cette fibre. Si l'une au moins des fibres de $\pi$ est transverse à $\mathcal{F}$, le même argument qu'en 3.5 montre que l'ensemble des points où $\mathcal{F}$ et $\pi$ ne sont pas transverses est une réunion finie de feuilles compactes.

Dans le cas où toutes les fibres de $\pi$ sont tangentes à $\mathcal{F}$, le feuilletage $\mathcal{F}$ est l'image réciproque par $\pi$ d'un feuilletage de $T$ et la situation est donc complètement décrite. Supposons donc qu'il existe des fibres transverses. Si $x$ est un point de $G / \Gamma$, la fibre de $\pi$ contenant $x$ est un espace homogène de $\widetilde{H}$ de la forme $\widetilde{H} / \Gamma_{x}$ où $\Gamma_{x}=x \Gamma x^{-1}$. La trace $\mathcal{F}_{x}$ de $\mathcal{F}$ sur cette fibre est donnée par l'action d'un sous- groupe fermé $F_{x} \subset \widetilde{H}$ de codimension 1 .

Commençons par étudier une telle fibre. 
Feuilletages holomorphes de codimension un sur les espaces homogènes complexes

Lemme 5.3. - Si $F_{x}$ est de type 2 ou 3, toutes les feuilles de $\mathcal{F}_{x}$ sont denses. Si $F_{x}$ est de type 1, les adhérences de toutes les feuilles sont des sous-variétés réelles de codimension 0,1 ou 2 .

Démonstration. - D'après le théorème de Lie, on dispose d'un morphisme surjectif $\sigma_{x}$ de $\tilde{H}$ dans $\mathbb{C}, \operatorname{Aff}(\mathbb{C})$ ou $\operatorname{PSL}(2, \mathbb{C})$ suivant le type, dont le noyau est l'intersection des conjugués de $F_{x}$ dans $\widetilde{H}$. Le relevé de $\mathcal{F}_{x}$ à $\widetilde{H}$ est défini par une fibration $p_{x}$ de $\widetilde{H}$ au-dessus de $\mathbb{C}$ ou $\mathbb{C P}^{1}$ et on a, pour $h$ dans $\tilde{H}$ et $\gamma$ dans $\Gamma_{x}$ :

$$
p_{x}(h \gamma)=\sigma_{x}\left(\gamma^{-1}\right)\left(p_{x}(h)\right) .
$$

La densité des feuilles de $\mathcal{F}_{x}$ est donc équivalente à celle des orbites de $\sigma_{x}\left(\Gamma_{x}\right)$ agissant sur $\mathbb{C}$ ou $\mathbb{C P}^{1}$.

Observons que le fait que $\Gamma_{x}$ soit cocompact dans $\widetilde{H}$ et que $\sigma_{x}$ soit surjectif montre que l'adhérence de $\sigma_{x}\left(\Gamma_{x}\right)$ est cocompacte dans $\mathbb{C}, \operatorname{Aff}(\mathbb{C})$ ou $\mathrm{SL}(2, \mathbb{C})$ respectivement.

Examinons d'abord le cas où $F_{x}$ est de type 1 , c'est-à-dire où $\sigma_{x}$ est à valeurs dans $\mathbb{C}$. Dans ce cas, l'adhérence de $\sigma_{x}\left(\Gamma_{x}\right)$ est un sous-groupe fermé de $\mathbb{C}$ dont les orbites sont donc, ou bien discrètes, ou bien $\mathbb{C}$ tout entier, ou bien une réunion discrète de droites. Il s'agit bien de sous-variétés réelles de codimension 0,1 ou 2. Les exemples des feuilletages linéaires des tores montrent que ces trois cas sont effectivement possibles.

Dans le cas 2, il n'est pas difficile de faire la liste des sous-groupes fermés de $\operatorname{Aff}(\mathbb{C})$ qui sont cocompacts. Outre $\operatorname{Aff}(\mathbb{C})$ tout entier, il s'agit simplement des sous-groupes formés des homothéties dont le coefficient est dans un sous-groupe cocompact de $\mathbb{C}^{*}$. De tels sous-groupes opèrent transitivement sur $\mathbb{C}$.

Enfin, dans le cas 3, on fait de même la liste des sous-groupes fermés cocompacts de $\operatorname{PSL}(2, \mathbb{C})$; ils sont discrets ou coïncident avec $\operatorname{PSL}(2, \mathbb{C})$. Leurs orbites sur la sphère de Riemann $\mathbb{C P}^{1}$ sont denses.

Nous étudions maintenant comment se comparent deux fibres transverses.

Lemme 5.4. - Si $x$ et $y$ sont deux points de $T$ tels que $\mathcal{F}$ soit transverse aux fibres $\pi^{-1}(x)$ et $\pi^{-1}(y)$, les traces de $\mathcal{F}$ sur ces deux fibres sont conjuguées par un difféomorphisme de classe $C^{\infty}$ transversalement holomorphe.

Démonstration. - L'ensemble des points de $T$ dont l'image inverse par $\pi$ est contenue dans une feuille compacte est un ensemble analytique qui 


\section{Étienne Ghys}

ne disconnecte donc pas $T$. On peut donc choisir un chemin lisse $\ell$ plongé dans $T$, joignant $x$ et $y$ et tel que $\mathcal{F}$ et $\pi$ sont transverses au-dessus de $\ell$. On contruit alors un champ de droites (de classe $C^{\infty}$ ) dans $\pi^{-1}(\ell)$ tangent à $\mathcal{F}$ et transverse aux fibres de $\pi$. Ceci permet de définir, par relèvement horizontal, une monodromie qui est un difféomorphisme de classe $C^{\infty}$ entre $\pi^{-1}(x)$ et $\pi^{-1}(y)$ et qui respecte les traces de $\mathcal{F}$. Ce difféomorphisme est de classe $C^{\infty}$ et il est transversalement holomorphe.

En particulier, les fibres transverses rencontrent toutes les feuilles non compactes. Toutes les feuilles non compactes s'accumulent donc sur toutes les feuilles compactes.

Un biholomorphisme global de $\mathbb{C}$ (resp. $\mathbb{C P}^{\mathbf{1}}$ ) est affine (resp. projectif). On voit donc que toutes les fibres transverses sont de même type. On dira que le feuilletage est de type 1,2 ou 3. Dans le cas 1 ou 2, la restriction du feuilletage à l'ouvert $U$ des feuilles non compactes est bien sûr un feuilletage transversalement affine complexe et, dans le cas 3, c'est un feuilletage transversalement projectif. La structure transverse globale de la restriction de $\mathcal{F}$ à $U$ est facile à décrire : il s'agit d'un groupe de difféomorphismes affines ou projectifs globaux de $\mathbb{C}$ ou $\mathbb{C P}^{1}$ engendré par $\sigma_{x_{0}}\left(\Gamma_{x_{0}}\right)\left(x_{0}\right.$ est un point base) d'une part et par l'action transverse des difféomorphismes de monodromie donnés dans 5.4. Par le même argument que dans 5.3, on voit que les adhérences des feuilles dans $U$ sont des sous-variétés de codimension réelle 2, 1 ou 0 . Dans le premier cas, les feuilles sont toutes fermées dans $U$ et définissent une fibration de $U$ au-dessus d'une courbe elliptique. Dans le second cas, les adhérences des feuilles de $U$ définissent une fibration sur le cercle. Enfin, dans le troisième cas, les feuilles sont toutes denses dans $U$.

Notons en particulier qu'il ne peut exister de feuille fermée dans $U$ que si la restriction du feuilletage à $U$ est donnée par une fibration au-dessus d'une courbe elliptique. Si $U$ n'est pas la variété $G / \Gamma$ toute entière, on voit donc que $U$ ne contient aucune feuille compacte. Si $U=G / \Gamma$, le feuilletage ne possède pas non plus de feuille compacte sauf s'il est défini par une fibration de $G / \Gamma$ au-dessus d'une courbe elliptique.

On remarquera aussi qu'un feuilletage transversalement affine peut toujours être considéré comme transversalement projectif.

Résumons notre discussion en donnant le théorème suivant.

THÉORÈME 5.5.- Soit $\mathcal{F}$ un feuilletage holomorphe de codimension 1 sur une variété compacte de la forme $G / \Gamma$ avec $\Gamma$ discret. Alors, il existe une fibration $\pi$ de $G / \Gamma$ sur un tore complexe $T$ dont les fibres sont des espaces 
Feuilletages holomorphes de codimension un sur les espaces homogènes complexes

homogènes de la forme $\widetilde{H} / \widetilde{H} \cap \Gamma$ avec $\widetilde{H}$ distingué dans $G$. Deux cas sont possibles:

- les fibres de $\pi$ sont contenues dans les feuilles de $\mathcal{F}$ de sorte que $\mathcal{F}$ est l'image réciproque par $\pi$ d'un feuilletage de $T$;

- le feuilletage $\mathcal{F}$ possède un nombre fini de feuilles compactes qui sont réunion de fibres de $\pi$; dans l'ouvert complémentaire, $\mathcal{F}$ est transverse aux fibres de $\pi$; les traces de $\mathcal{F}$ sur les fibres $\pi^{-1}(x)$ transverses sont des feuilletages homogènes, c'est-à-dire donnés par l'action d'un sousgroupe $F_{x}$ de codimension 1 dans $\tilde{H}$ sur l'espace $\tilde{H} / \tilde{H} \cap \Gamma$; dans cet ouvert, le feuilletage est transversalement projectif et les adhérences des feuilles sont des sous-variétés réelles de codimension 0,1 ou 2.

\section{Tourbillonnements}

Dans cette section, nous étudions le cas où le feuilletage considéré sur $G / \Gamma$ possède des feuilles compactes, c'est-à-dire où certaines fibres de la fibration $\pi$ sont contenues dans une feuille de $\mathcal{F}$. En fait, nous nous contenterons de quatre exemples qui illustrent les possibilités.

Exemple 6.1. - Nous avons construit des feuilletages sur des tores complexes qui possèdent des feuilles compactes et qui sont bien sûr de type 1 .

Exemple 6.2.- Considérons le groupe de Lie complexe $G$ de dimension 3 produit semi-direct de $\mathbb{C}^{2}$ par $\mathbb{C}^{*}$ où l'élément $z$ de $\mathbb{C}^{*}$ opère sur $\mathbb{C}^{2}$ par : $z \cdot(x, y)=\left(z x, z^{-1} y\right)$. L'algèbre de Lie est engendrée par les trois champs de vecteurs :

$$
X=z \frac{\partial}{\partial x}, \quad Y=z^{-1} \frac{\partial}{\partial y}, \quad Z=z \frac{\partial}{\partial z} .
$$

Les relations de crochet sont

$$
[X, Y]=0, \quad[X, Z]=-X, \quad[Y, Z]=Y .
$$

Il existe de nombreux sous-groupes discrets cocompacts de ce groupe construits de la façon suivante. Soit $\Lambda$ un réseau de $\mathbb{C}^{2}$ invariant par l'action d'un certain $\zeta$ de $\mathbb{C}^{*}$ de module différent de 1 . Alors le sous-groupe engendré par $\Lambda \in \mathbb{C}^{2}$ et $\zeta \in \mathbb{C}^{*}$ est un sous-groupe discret cocompact de $\Gamma$ de $G$. La variété $M=G / \Gamma$ est un fibré en tores $\mathbb{C}^{2} / \Lambda$ au-dessus de la courbe elliptique 
$C$, quotient de $\mathbb{C}^{*}$ par les puissances de $\zeta$. On pourra consulter [3] pour une description de ces exemples.

Le sous-espace engendré par $X$ et $Z$ est une sous-algèbre et définit donc un feuilletage homogène de codimension 1 sur $M$ (de type 2). Considérons maintenant une fonction holomorphe $u$ de $C$ vers la droite projective constituée des droites contenues dans la sous-algèbre engendrée par $Y$ et $Z$. On note encore $u$ la fonction holomorphe sur $M$ obtenue en composant avec la projection de $M$ sur $C$. En chaque point $m$ de $M$, on peut donc considérer l'hyperplan engendré par $X$ et la droite $u(m)$. Les relations de crochet ci-dessus montrent que ceci est intégrable. Pour chaque fonction méromorphe sur la courbe elliptique $C$, on obtient donc un feuilletage holomorphe sur $M$. Lorsque la fonction méromorphe est non constante, la projection $\pi$ construite à la section 5 dans le cas général est, dans cet exemple, la projection de $M$ sur $C$. Les fibres de $\pi$ sont des tores complexes $\mathbb{C}^{2} / \Lambda$. Les fibres qui ne sont pas transverses au feuilletage sont celles où la fonction $u$ prend comme valeur la droite engendrée par $Y$; ce sont des feuilles compactes (qui sont des tores). Les fibres transverses, quant à elles, sont simplement des tores sur lesquels le feuilletage induit est linéaire, donc de type 1 . Sur l'ouvert complémentaire aux feuilles compactes, le feuilletage est transversalement affine.

Exemple 6.3. - Le groupe affine $\operatorname{Aff}(\mathbb{C})$ supporte deux formes différentielles holomorphes invariantes à droite $\omega$ et $\alpha$ vérifiant les relations suivantes :

$$
\mathrm{d} \omega=\omega \wedge \alpha, \quad \mathrm{d} \alpha=0 .
$$

Le sous-groupe des homothéties a pour équation $\omega=0$.

Si $F$ est un sous-groupe de type 2 d'un groupe de Lie $H$ et si $\Gamma$ est un sous-groupe discret cocompact de $H$, il existe deux formes naturelles $\omega$ et $\alpha$ sur $H / \Gamma$ et le feuilletage d'équation $\omega=0$ est le feuilletage homogène dont les feuilles sont les orbites de $F$. Soit $C=\mathbb{C} / \Lambda$ une courbe elliptique et $u: C \rightarrow \mathbb{C P}^{1}$ une fonction méromorphe. Nous allons construire un feuilletage sur $G / \Gamma \times \mathbb{C} / \Lambda$. Considérons la forme différentielle méromorphe suivante sur $G / \Gamma \times \mathbb{C} / \Lambda$ :

$$
\Omega=\omega+u \alpha+\mathrm{d} u
$$

(nous avons confondu $\omega$ et la forme image réciproque dans le produit). Il est facile de vérifier que $\Omega$ est intégrable. De même que nous l'avons fait pour les tores, on voit que le feuilletage qu'elle définit se prolonge en fait 
Feuilletages holomorphes de codimension un sur les espaces homogènes complexes

en un feuilletage holomorphe non singulier dont les feuilles compactes sont les produits de $H / \Gamma$ par les pôles de $u$, sur lesquelles spiralent les autres feuilles. Dans cet exemple, le tore de base $T$ est la courbe elliptique $C$, les traces du feuilletage sur les fibres transverses sont toutes conjuguées entre elles et sont de type 2, à feuilles denses. Le feuilletage, dans l'ouvert des feuilles non compactes, est transversalement affine.

Exemple 6.4.- Nous illustrons sur un exemple le fait qu'il n'est pas toujours possible de faire tourbillonner un feuilletage. Soit $\Gamma$ un sousgroupe discret cocompact de $\operatorname{SL}(2, \mathbb{C})$ et $\Lambda$ un réseau dans $\mathbb{C}$. Nous allons décrire complètement tous les feuilletages holomorphes de codimension 1 sur le produit $M=\mathrm{SL}(2, \mathbb{C}) / \Gamma \times \mathbb{C} / \Lambda$. On note $\pi$ la projection sur le second facteur. Si $\mathcal{F}$ est un tel feuilletage qui n'est pas la fibration de $M$ sur la courbe elliptique $C=\mathbb{C} / \Lambda$, nous savons que pour $x$ hors d'un ensemble fini de $\Sigma$ de $C$, la trace de $\mathcal{F}$ sur $\operatorname{SL}(2, \mathbb{C}) / \Gamma \times\{x\} \simeq$ $\mathrm{SL}(2, \mathbb{C}) / \Gamma$ est donnée par l'action d'un sous-groupe $H_{x}$ de codimension 1 dans $\mathrm{SL}(2, \mathbb{C})$. Remarquons que les sous-groupes de codimension 1 dans $\mathrm{SL}(2, \mathbb{C})$ sont naturellement paramétrés par la droite projective de $\mathbb{C P}^{1} ;$ ce sont les stabilisateurs des divers points de $\mathbb{C P}^{1}$ dans l'action projective de $\mathrm{SL}(2, \mathbb{C})$ ou encore les conjugués du sous-groupe des matrices triangulaires supérieures. L'application $\tau$ qui associe à $x$ de $C \backslash \Sigma$ le sous-groupe $F_{x}$ est donc une fonction holomorphe à valeurs dans $\mathbb{C P}^{1}$.

Soit $V$ un ouvert de $C$ et $u: V \rightarrow \mathrm{SL}(2, \mathbb{C})$ une fonction holomorphe. Considérons le biholomorphisme de $\Psi_{u}$ de $\pi^{-1}(V)$ envoyant le point $(y, x)$ sur $(u(x) \cdot y, x)$. Soit $\mathcal{F}_{0}$ le feuilletage de codimension 1 sur $M=\operatorname{SL}(2, \mathbb{C}) / \Gamma \times C$ produit par $C$ du feuilletage de $\mathrm{SL}(2, \mathbb{C}) / \Gamma$ dont les feuilles sont les orbites du sous-groupe $B$ des matrices triangulaires. L'image de $\mathcal{F}_{0}$ par $\Psi_{u}$ est un feuilletage $\mathcal{F}_{u}$ de $\pi^{-1}(V)$ qui rencontre transversalement les fibres $\pi^{-1}(x)$ sur le feuilletage donné par les orbites de $H_{x}$ où $H_{x}$ est le conjugué par $u(x)$ de $B$. Il est clair que si l'on remplace $u$ par une autre fonction holomorphe $c: V \rightarrow \mathrm{SL}(2, \mathbb{C})$ telle que les projections de $u$ et $v$ dans $\mathbb{C P}^{1}=\mathrm{SL}(2, \mathbb{C}) / B$ sont les mêmes, les feuilletages $\mathcal{F}_{u}$ et $\mathcal{F}_{v}$ coïncident. Partant d'une application méromorphe non constante $\tau: C \rightarrow \mathbb{C P}^{1}$, on ne peut pas trouver d'application holomorphe $u: C \rightarrow \mathrm{SL}(2, \mathbb{C})$ dont la projection dans $\mathbb{C P}^{1}$ soit $\tau$ mais on peut le faire localement. Plus précisément, on peut trouver deux ouverts $V_{1}$ et $V_{2}$ de $C$ et deux fonctions holomorphes $u_{1}$ et $u_{2}$ définies sur $V_{1}$ et $V_{2}$, à valeurs dans $\mathrm{SL}(2, \mathbb{C})$, qui relèvent $\tau$ sur $V_{1}$ et $V_{2}$. Les difféomorphismes correspondants $\Psi_{u_{1}}$ et $\Psi_{u_{2}}$ ne coïncident pas sur l'intersection de leurs domaines de définition $\pi^{-1}\left(V_{1}\right) \cap \pi^{-1}\left(V_{2}\right)$ mais les feuilletages 
$\mathcal{F}_{u_{1}}$ et $\mathcal{F}_{u_{2}}$ coïncident. Par conséquent, nous voyons que pour toute application holomorphe $\tau: C \rightarrow \mathbb{C P}^{1}$, il existe un feuilletage holomorphe sur $\mathrm{SL}(2, \mathbb{C}) / \Gamma \times C$ transverse à toutes les fibres de $\pi$ et qui intersecte $\pi^{-1}(x)$ sur les orbites de $\tau(x) \in \mathbb{C P}^{1}$, considéré comme un sous-groupe de codimension 1 de $\mathrm{SL}(2, \mathbb{C})$.

Nous nous proposons de montrer qu'on obtient ainsi tous les feuilletages holomorphes de $\mathrm{SL}(2, \mathbb{C}) / \Gamma \times C$ (autres que la fibration $\pi$ ). En particulier, nous allons montrer qu'un tel feuilletage est transverse à toutes les fibres de $\pi$, c'est-à-dire que l'ensemble $\Sigma$ est vide.

LEMME 6.5. - L'application $\tau$ se prolonge en une application holomorphe définie sur $C$ tout entier et à valeurs dans $\mathbb{C P}^{1}$.

Démonstration. - Soit $F$ un sous-groupe de codimension 1 de $\mathrm{SL}(2, \mathbb{C})$. L'ensemble des points $m$ de $M$ tels que l'orbite de $m$ par $F$ est tangente en $m$ à $\mathcal{F}$ est un ensemble analytique de $M$, contenant évidemment $\pi^{-1}(\Sigma)$. L'application $\tau: C \backslash \Sigma \rightarrow \mathbb{C P}^{1}$, si elle n'est pas constante, a donc la propriété que la réunion de $\Sigma$ et de chacune de ses fibres est un ensemble analytique (donc fini). Ceci montre que $\tau$ n'a pas de singularité essentielle et se prolonge holomorphiquement à $C$ tout entier.

Ainsi, même si $x$ est dans $\Sigma$, nous pouvons définir un sous-groupe $H_{x}$ de codimension 1 dans $\mathrm{SL}(2, \mathbb{C})$.

Nous allons modifier localement un feuilletage donné par un biholomorphisme pour rendre $\tau$ constant. Un point de $C$ étant donné (même dans $\Sigma$ ), on peut trouver un voisinage ouvert $V$ de ce point et une fonction holomorphe $u: V \rightarrow \mathrm{SL}(2, \mathbb{C})$ tels que, pour $x$ dans $V$, le conjugué de $B$ par $u(x)$ soit $H_{x}$. Étudions l'image $\mathcal{F}^{\prime}$ de $\mathcal{F}$ (restreint à $u^{-1}(U)$ ) par le biholomorphisme $\Psi_{u}^{-1}$ et montrons que ce n'est autre que le feuilletage produit $\mathcal{F}_{0}$. Il en résultera que $\mathcal{F}^{\prime}$ et donc $\mathcal{F}$ sont partout transverses aux fibres de $\pi$, c'est-à-dire que $\Sigma$ est vide, comme annoncé.

L'équation de structure du dual de l'algèbre de Lie sur $\operatorname{SL}(2, \mathbb{C})$ est donnée par trois formes holomorphes invariantes à droite $\omega, \alpha$ et $\omega^{\prime}$ telles que :

$$
\mathrm{d} \omega=\omega \wedge \alpha, \quad \mathrm{d} \omega^{\prime}=-\omega^{\prime} \wedge \alpha, \quad \mathrm{d} \alpha=\omega \wedge \omega^{\prime} .
$$

Le sous-groupe $B$ a pour équation $\omega=0$. Ces trois formes définissent des formes notées de la même façon sur le quotient $\operatorname{SL}(2, \mathbb{C}) / \Gamma$ et sur le produit $M=\operatorname{SL}(2, \mathbb{C}) / \Gamma \times \mathbb{C} / \Lambda$. Cherchons à écrire l'équation du feuilletage $\mathcal{F}^{\prime}$. 
Feuilletages holomorphes de codimension un sur les espaces homogènes complexes

Nous savons d'une part que la trace de $\mathcal{F}^{\prime}$ sur $\pi^{-1}(x)$ avec $x \notin \Sigma$ est donnée par les orbites de $B$ et a donc pour équation $\omega=0$. Par ailleurs, nous savons que le plan tangent à $\mathcal{F}$ est constant sur les fibres de $\pi$, par rapport à la trivialisation canonique du fibré tangent à $M$. Étant donnée la nature de $\Psi_{u}$, cette même propriété est satisfaite pour $\mathcal{F}^{\prime}$. En d'autres termes, $\mathcal{F}^{\prime}$ est défini dans $\pi^{-1}(V \backslash \Sigma)$ par une équation de la forme :

$$
\Omega=\omega+f(x) \mathrm{d} z
$$

où $z$ est une coordonnée locale dans $V$ et $f$ est une fonction méromorphe dans $V$ qui a des pôles sur $\Sigma$. En écrivant que $\Omega$ est intégrable et en utilisant les formules ci-dessus, on obtient que $f$ est nécessairement identiquement nulle. Il en résulte en particulier que $f$ n'a pas de pôle, c'est-à-dire que $\Sigma$ est vide mais aussi que $\mathcal{F}^{\prime}$ cö̈ncide avec le feuilletage produit $\mathcal{F}_{0}$, comme nous l'avions annoncé. Si deux feuilletages de $M$ ont la même fonction $\tau$, nous voyons donc qu'après une transformation par le même difféomorphisme (local) $\Psi_{u}$, ils coïncident. C'est donc que deux feuilletages ayant la même fonction $\tau$ sont les mêmes. Résumons notre discussion qui montre un cas où le tourbillonnement est impossible.

THÉORÈME 6.6. - Soit $\Gamma$ un sous-groupe discret cocompact de $\mathrm{SL}(2, \mathbb{C})$. Identifions l'espace des sous-groupes de codimension 1 de $\mathrm{SL}(2, \mathbb{C}) \grave{a} \mathbb{C P}^{1}$. Soit $C$ une courbe elliptique et $\tau: C \rightarrow \mathbb{C P}^{1}$ une fonction méromorphe sur $C$. Il existe un unique feuilletage holomorphe $\mathcal{F}_{\tau}$ sur le produit $\mathrm{SL}(2, \mathbb{C}) / \Gamma \times$ $C$ qui intersecte transversalement $\mathrm{SL}(2, \mathbb{C}) / \Gamma \times\{x\}$ sur les orbites du sousgroupe $\tau(x)$. Tout feuilletage holomorphe de codimension 1 sur $\mathrm{SL}(2, \mathbb{C}) / \Gamma \times$ $C$ autre que la fibration au-dessus de $C$ est l'un de ces feuilletages $\mathcal{F}_{\tau}$.

\section{Quelques exemples dans le cas général}

Les techniques que nous avons développées nous permettent une bonne description des feuilletages de codimension 1 sur les variétés homogènes compactes quelconques. Nous ne chercherons pas les énoncés les plus précis qui nous entraîneraient dans de longues listes de cas particuliers qui n'auraient pas d'intérêt. Nous nous contenterons de démontrer le théorème 1.4 et d'étudier deux exemples.

Soit $\mathcal{F}$ un feuilletage holomorphe de codimention 1 sur la variété homogène compacte $M=G / H$. En chaque point de $M$, l'espace tangent à $M$ est 
un quotient de l'algèbre de Lie $\mathcal{G}$ de $G$. L'espace tangent à $\mathcal{F}$ permet donc de définir une application holomorphe $\Phi$ de $M$ vers l'espace $Y$ des hyperplans de $\mathcal{G}$, c'est-à-dire vers un espace projectif. On peut donc appliquer le corollaire 2.2 : il existe un sous-groupe complexe fermé $\widetilde{H}$ contenant $H$ et une application holomorphe $\Phi^{\prime}: G / \widetilde{H} \rightarrow Y$ tels que :

- $\Phi=\Phi^{\prime} \circ \pi$ où $\pi$ désigne la projection de $G / H$ sur $G / \widetilde{H}$;

- les fibres de $\Phi^{\prime}$ sont finies.

Autrement dit, il existe une fibration $\pi$ de $M$ au-dessus de la variété kählérienne $G / \widetilde{H}$; les fibres de $\pi$ sont des espaces homogènes $\tilde{H} / H$ et l'application $\Phi$ est constante sur ces fibres. Comme précédemment, il en résulte que l'image réciproque de $\mathcal{F}$ dans $G$ est invariante par les translations à droite par $\widetilde{H}$. Les fibres de $\pi$ non transverses à $\mathcal{F}$ sont donc contenues dans des feuilles compactes. La restriction de $\mathcal{F}$ à une fibre transverse $\pi^{-1}(x)$ est donnée par l'action d'un sous-groupe de $F_{x}$ de codimension 1 dans $\widetilde{H}$.

Nous sommes donc dans une situation très proche de celle étudiée à la section 5. Les seules différences sont que la base de $\pi$ n'est plus nécessairement un tore et que les fibres transverses sont maintenant des espaces homogènes $\widetilde{H} / H$ où $H$ n'est pas nécessairement discret. La restriction de $\mathcal{F}$ à une fibre transverse $\pi^{-1}(x)$ est cependant encore donnée par l'action d'un sous-groupe $F_{x}$ de codimension 1 dans $\widetilde{H}$ et nous dirons encore qu'il s'agit d'un feuilletage homogène.

Il est bien sûr possible que le feuilletage soit partout transverse à $\pi$. Dans ce cas, il s'agit d'un feuilletage transversalement projectif qui ne peut posséder de feuilles compactes que si toutes le sont; le feuilletage est alors une fibration au-dessus d'une courbe elliptique.

On remarquera que les preuves des lemmes 5.3 et 5.4 s'étendent sans difficulté à ce cas.

On obtient donc le théorème suivant, plus fort que le théorème 1.4.

THÉORÈME 7.1. - Soit $\mathcal{F}$ un feuilletage holomorphe sur une variété complexe compacte homogène $M$. Il existe une fibration $\pi$ de $M$ sur une variété kählérienne homogène $K$ et l'une des propriétés suivantes est vérifiée :

- le feuilletage est l'image réciproque d'un feuilletage de $K$;

- le feuilletage possède un nombre fini de feuilles compactes qui sont des réunions de fibres de $\pi$; dans l'ouvert complémentaire, toutes les fibres 
Feuilletages holomorphes de codimension un sur les espaces homogènes complexes

de $\pi$ sont transverses à $\mathcal{F}$ et la trace de $\mathcal{F}$ sur ces fibres est un feuilletage homogène; dans cet ouvert, le feuilletage est transversalement projectif et les adhérences des feuilles sont des sous-variétés qui sont simultanément de codimension 0,1 ou 2 ; toutes les feuilles compactes sont adhérentes à toutes les feuilles non compactes.

Pour illustrer ce théorème, nous donnons une construction très générale.

Exemple 7.2. - Considérons le feuilletage $\mathcal{L}$ de $\mathbb{C}^{n+1}$ dont les feuilles sont les hyperplans parallèles à une direction donnée. Soit $V$ une variété complexe compacte quelconque et $u: V \rightarrow \mathbb{C P}^{n}$ une application holomorphe (qui n'est pas nécessairement un plongement). Soit $\bar{V}$ l'image réciproque par $u$ du fibré canonique au-dessus de $\mathbb{C P}^{n}$. C'est un fibré en droites audessus de $V$ et on dispose d'une application holomorphe naturelle $\bar{u}$ de $\bar{V}$ vers $\mathbb{C}^{n+1}$ qui envoie les fibres sur les droites qui passent par l'origine et qui envoie la section nulle sur l'origine. En choississant la direction de l'hyperplan génériquement, on peut faire en sorte que $\bar{u}$ soit transverse à $\mathcal{L}$ sur le complémentaire de la section nulle, et on obtient ainsi un feuilletage holomorphe $\overline{\mathcal{L}}$ sur ce complémentaire, évidemment invariant par les homothéties dans les fibres. Si l'on choisit un nombre complexe non nul $\zeta$, de module différent de 1 , et si on quotiente le complémentaire de 0 dans chaque fibre de $\bar{V}$ par l'homothétie de rapport $\zeta$, on obtient une variété compacte $\widetilde{V}$ fibrée en courbes elliptiques au-dessus de $V$ et munie d'un feuilletage $\tilde{\mathcal{L}}$. Dans de nombreux cas, la variété $\widetilde{V}$ est homogène. La dynamique de ces exemples est assez pauvre : les feuilles non compactes spiralent sur les feuilles compactes. La géométrie de ces feuilletages est cependant très riche : les feuilles compactes sont des fibrés en courbes elliptiques au-dessus de l'image inverse par $u$ d'un hyperplan et on peut donc construire des exemples où des feuilles compactes sont compliquées.

Exemple 7.3.- Pour terminer cet article, nous traitons du cas des variétés de Hopf. Soit $M$ le quotient de $\mathbb{C}^{n} \backslash\{0\}$ par une homothétie de rapport $\zeta$ (non nul et de module différent de 1 ). C'est un espace homogène sous l'action de $\mathrm{SL}(n, \mathbb{C})$, fibré en courbes elliptiques au-dessus de l'espace projectif $\mathbb{C P}^{n-1}$. Nous allons déterminer tous les feuilletages holomorphes $\mathcal{F}$ de codimension 1 sur $M$. Appliquons la méthode générale. Le relevé $\widetilde{\mathcal{F}}$ de $\mathcal{F}$ à $\mathbb{C}^{n} \backslash\{0\}$ permet de déterminer une application holomorphe $\widetilde{\Phi}$ de $\mathbb{C}^{n} \backslash\{0\}$ vers l'espace projectif $\left(\mathbb{C P}^{n-1}\right)^{*}$ des hyperplans de $\mathbb{C}^{n}$, évidemment invariante par l'homothétie de rapport $\zeta$, et qui produit donc une application holomorphe $\Phi$ de $M$ vers $\left(\mathbb{C P}^{n-1}\right)^{*}$. Puisque la dimension 
de $M$ est strictement supérieure à $n-1, \Phi$ ne peut être à fibres finies. Il est facile de déduire de 2.2 que $\Phi$ s'obtient en composant la projection de $M$ sur $\mathbb{C P}^{n-1}$ et une application holomorphe (i.e. rationnelle) de $\mathbb{C P}^{n-1}$ vers $\left(\mathbb{C P}^{n-1}\right)^{*}$. Cela signifie précisément que $\widetilde{\mathcal{F}}$ est défini par une forme holomorphe $\omega$ de $\mathbb{C}^{n}$ qui est homogène d'une certain degré $k$, intégrable, et dont la seule singularité est à l'origine.

Distinguons deux cas.

Si la dimension $n$ est au moins 3 , la forme $\omega$ possède une intégrale première qui est un polynôme homogène de degré $k+1$ sur $\mathbb{C}^{n}$ (voir par exemple [10]). Réciproquement, les surfaces de niveau d'un polynôme homogène à singularité isolée définissent un feuilletage holomorphe non singulier sur $\mathbb{C}^{n} \backslash\{0\}$ qui passe au quotient sur $M$. Ces feuilletages possèdent une feuille compacte sur laquelle spiralent les autres feuilles. On pourra consulter [11] pour une description de leur géométrie.

Si la dimension $n$ est égale à $2, \omega$ est une forme homogène à singularité isolée quelconque dans $\mathbb{C}^{2}$. Les feuilletages obtenus sur les surfaces de Hopf associées ont des feuilles compactes qui sont des courbes elliptiques correspondant aux séparatrices de $\omega$; elles sont en nombre fini sauf si le feuilletage a toutes ses feuilles compactes.

\section{Remerciements}

Je voudrais remercier Dominique Cerveau dont une question est à l'origine de cet article. J'ai par ailleurs bénéficié de conversations très utiles avec Michel Brion et Bruno Sévennec.

\section{Bibliographie}

[1] Bотт (R.) .- Lectures on characteristic classes and foliations, Notes by L. Conlon, Mexico 1971, Lecture Notes in Mathematics, Springer-Verlag 279 (1973).

[2] Bott (R.) et TU (L. W.) .- Differential forms in Algebraic Topology, Graduate text in Mathematics, Springer-Verlag 82 (1982).

[3] Ghys (E.) et Verjovsky (A.) . - Locally free holomorphic actions of the complex affine group, Proceedings of Geometric Study of Foliations, Tokyo novembre 1993, éd. T. Mizutani et al., World Scientific Singapore (1994), pp. 201-217.

[4] Greub (W.), Halperin (S.) et Vanstone (R.) .- Connections, curvature and cohomology, Academic Press 3 (1976).

[5] HeCtor (G.) .- Architecture des feuilletages de classe $C^{2}, 3^{\text {ème }}$ rencontre de géométrie du Schnepfenried, Vol. 1, Astérique 107-108 (1992), pp. 243-258. 
Feuilletages holomorphes de codimension un sur les espaces homogènes complexes

[6] HUCKLEBERRY (A. T.) .- Actions of groups of holomorphic transformations, in Encyclopaedia of Mathematical Sciences, Several complex variables, W. Barth et R. Narasimhan (éds), Springer-Verlag 6 (1990).

[7] Jouanolou (J.-P.) .- Équations de Pfaff algébriques, Springer Lecture Notes in Maths. 708 (1979).

[8] Jounalou (J.-P.) .- Feuilles compactes des feuilletages algébriques, Math. Ann. 241 (1979), pp. 69-72.

[9] LIE (S.) .- Theorie der Transformationsgruppen, Math. Ann. 6 (1880).

[10] Malgrange (B.) .- Frobenius avec singularités. 1. Codimension 1, Publ. I.H.E.S. 46 (1976), pp. 163-173.

[11] MiLnOR (J.) . - Singular points of complex hypersurfaces, Ann. of Mathematical Studies 61, Princeton University Press (1968).

[12] Montgomery (D.) .- Simply connected homogeneous spaces, Proc. A.M.S. 1 (1950), pp. 457-569.

[13] Sullivan (D.) . - Seminar on conformal and hyperbolic geometry, Notes par M. Baker et J. Seade, I.H.E.S. (1982). 